\title{
Sevoflurane exerts improved protective effects than propofol on hypoxia-reoxygenation injury by regulating the microRNA-221-5p/ADAM8 axis in cardiomyocytes
}

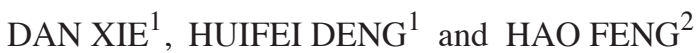 \\ ${ }^{1}$ Department of Anesthesiology, Affiliated Hospital of North Sichuan Medical College; \\ ${ }^{2}$ Department of Neurosurgery, Nanchong Central Hospital, The Second Clinical Medical College, \\ North Sichuan Medical College, Nanchong, Sichuan 637000, P.R. China
}

Received October 20, 2020; Accepted May 24, 2021

DOI: $10.3892 /$ etm.2021.10325

\begin{abstract}
Myocardial ischemia-reperfusion (I/R) injury is a leading cause of heart disease and death. Decreasing the detrimental effect of $\mathrm{I} / \mathrm{R}$ remains an urgent issue in clinical practice. The present study examined the interaction of the anesthetics (sevoflurane and propofol), ADAM8, and microRNA (miR)-221-5p in myocardial tissue protection in the hypoxia-reoxygenation $(\mathrm{H} / \mathrm{R})$ model. H9C2 cells were cultured and subjected to $\mathrm{H} / \mathrm{R}$ stimulation for further verifications in vitro. Reverse transcription-quantitative PCR or western blotting was performed to evaluate mRNA or protein expression levels. Cell Counting Kit-8, BrdU, and caspase-3 activity assays were performed to investigate cell viability, proliferation and apoptosis. A dual-luciferase reporter assay was performed to verify the association between miR-221-5p and ADAM8. Sevoflurane had greater protective effects on the life of cardiomyocytes with $\mathrm{H} / \mathrm{R}$ injury compared with propofol by promoting cell viability, proliferation and inhibiting apoptosis. Concurrently, compared with propofol-treated $\mathrm{H} / \mathrm{R}$ injured cardiomyocytes, the expression level of ADAM8 in sevoflurane-treated $\mathrm{H} / \mathrm{R}$ injured cardiomyocytes was higher. In addition, overexpression of ADAM8 promoted the cell viability and proliferation of sevoflurane-treated cardiomyocytes with H/R injury but inhibited cell apoptosis, while the downregulation of miR-221-5p showed an opposite trend to that of ADAM8 overexpression. The present data provide evidence that sevoflurane can mediate the miR-221-5p/ADAM8 axis, playing a better protective role compared with propofol in cardiomyocytes with $\mathrm{H} / \mathrm{R}$ injury.
\end{abstract}

Correspondence to: Dr Hao Feng, Department of Neurosurgery, Nanchong Central Hospital, The Second Clinical Medical College, North Sichuan Medical College, 97 South Renmin Road, Nanchong, Sichuan 637000, P.R. China

E-mail: hao_feng66@163.com

Key words: sevoflurane, propofol, ADAM8, microRNA-221-5p, hypoxia-reoxygenation, cardiomyocytes

\section{Introduction}

Worldwide, myocardial infarction is one of the most common and severe heart diseases (1). During myocardial infarction, ischemia-reperfusion $(\mathrm{I} / \mathrm{R})$ can result in myocardial injury (2). In order to investigate the mechanisms of $I / R$ injury, myocardial hypoxia-reoxygenation $(\mathrm{H} / \mathrm{R})$ is an ideal in vitro model (3-5). Myocardial $\mathrm{H} / \mathrm{R}$ has been reported to have a complex association with a number of molecules that ultimately damage the heart $(6,7)$. In the past decade, despite some advances in therapeutic drugs that decrease myocardial I/R damage, new cardiac protective drugs are still urgently needed to improve treatment outcomes and minimize the injury caused by myocardial I/R.

Post-anesthesia adaptation is a form of cellular protection (8). Tissues are better able to resist I/R injury when they are treated with volatile anesthetics $(8,9)$. Propofol exerts a protective effect on I/R injury as an anesthetic (10). The mechanism of the protective effect was found to involve free radical scavenging (11) or inhibition of calcium channels (12). However, as a new type of anesthetic, sevoflurane, is widely applied in cardiac surgery because it acts faster, gives more stable recovery, and more efficient anesthesia (13). In addition, sevoflurane exhibits excellent cardioprotective performance in I/R injury (14). To better understand and utilize the protective effects of anesthetics, more research is still needed. Therefore, the aim of the present study was to determine whether sevoflurane exerts a better cardioprotective effect than propofol on $\mathrm{H} / \mathrm{R}$ injury in cardiomyocytes and the associated mechanism.

A disintegrin and metalloproteinase (ADAM) is a family of peptidase proteins regulating proteolysis and mediate cell-cell and cell-matrix interactions (15). As one of the key members, ADAM8 was originally revealed to encode a protein containing a carboxy-terminal transmembrane domain (16). Subsequent research revealed that rat heart transplantation-induced distal myocardial remodeling in I/R injury or myocardial infarction was associated with the upregulation of ADAM8 (17). According to RNA-seq analysis (GSE4386), ADAM8 is differently expressed in the samples treated with sevoflurane and propofol. Besides, GO enrichment suggested that ADAM8 is associated with cellular process and cell activation. Based on 
the findings of the aforementioned studies, the role of ADAM8 in cell viability was further investigated, when cardiomyocytes are undergoing $\mathrm{H} / \mathrm{R}$ injury.

MicroRNAs (miRNAs/miRs) are non-coding RNAs that mediate target gene expression by binding to the 3 ' untranslated region (3'-UTR). Several miRNAs have been identified to be associated with myocardial H/R injury. For example, a study revealed that miR-101a-3p suppressed H9C2 cell proliferation and, during H/R injury, promoted H9C2 cell apoptosis (18). Likewise, miR-142-p could promote cell apoptosis in a cardiomyocyte model of H/R injury (19). Upregulation of miR-221-5p was observed in myocardial hypertrophy and heart failure (20). The present study attempted to explore the role of miR-221-5p in influencing H9C2 cell proliferation and apoptosis, following $\mathrm{H} / \mathrm{R}$ injury. Interestingly, it was found that miR-221-5p could target ADAM8 and negatively affected the protection of $\mathrm{H} 9 \mathrm{C} 2$ cells subjected to H/R injury.

The present study aimed to determine the roles and interactions of sevoflurane, propofol, ADAM8 and miR-221-5p in the protection of myocardial tissue in the H/R model. The study outcomes will provide more information on the therapeutic strategy in the alleviation of the negative effects of $\mathrm{I} / \mathrm{R}$ on the ischemic myocardial tissue in clinical practice.

\section{Materials and methods}

Cell culture and H/R model. Rat embryo cardiomyocyte cell line (H9C2), obtained from American Type Culture Collection, was cultured in DMEM medium (Hyclone; Cytvia) with $10 \%$ fetal bovine serum (Gibco; Thermo Fisher Scientific, Inc.) and $1 \%$ penicillin/streptomycin (Invitrogen; Thermo Fisher Scientific, Inc.). The cells were cultured in an incubator with $5 \% \mathrm{CO}_{2}$ at $37^{\circ} \mathrm{C}$. The $\mathrm{H} / \mathrm{R}$ model was established by culturing $\mathrm{H} 9 \mathrm{C} 2$ cells in an oxygen-deficient condition $\left(95 \% \mathrm{~N}_{2}\right.$ and $5 \% \mathrm{CO}_{2}$ ) for $6 \mathrm{~h}$. Subsequently, the cells were placed back to the normal condition $\left(95 \% \mathrm{O}_{2}\right.$ and $\left.5 \% \mathrm{CO}_{2}\right)$ for another $6 \mathrm{~h}$.

Bioinformatics analysis. GSE4386 (https://www.ncbi.nlm. nih.gov/geo/query/acc.cgi?acc=GSE4386) downloaded from GEO comprised the mRNA microarray datasets including sevoflurane and propofol samples. With $\mathrm{P}<0.05$ and $\log$ fold change $(\mathrm{FC})>1$, the upregulated genes in sevoflurane samples were screened from GSE4386. Subsequently, STRING (https://string-db.org/) and Metascape (http://metascape. org/gp/index.html\#/main/step1) were used for biological process analysis of the upregulated genes. Finally, Venny 2.1.0 (http://bioinfogp.cnb.csic.es/tools/venny/) was used to examine the overlap of the common genes in STRING and Metascape.

Cell transfection and cell treatment. The ADAM8 overexpression vectors were obtained from GeneCreate Biotech. Briefly, the full length of ADAM8 was synthesized and subcloned into pcDNA3.1 vector (Invitrogen; Thermo Fisher Scientific, Inc.). The empty vector pcDNA 3.1 was used as the negative control. The small interfering RNA (siRNA) named si-ADAM8 (5'-CGGCACCUGCAUGACAACGUA-3') and non-targeting siRNA (si-NC, 5'-UUCUCCGAACGUGUCACGUTT-3') were designed and synthesized by Guangzhou RiboBio Co., Ltd. miR-221-5p mimic (sense, 5'-AGCUACAUUGUCUGC UGGGUUUC-3'), mimic-NC (5'-GAAAUGUACUUGAGC
GUGGAGAC-3'), miR-221-5p inhibitor (5'-ACAGAAAUC UACAUUGUAUGCCAGGU-3') and inhibitor-NC (5'-CUA AAACCGGCCGUACGGCGUU-3') were synthesized by Guangzhou RiboBio Co., Ltd. Cell transfection was carried out using Lipofectamine ${ }^{\mathrm{TM}} 3000$ Transfection Reagent (Thermo Fisher Scientific, Inc.), following the manufacturer's instructions.

For the treatment of sevoflurane $(\mathrm{S})$ and propofol $(\mathrm{P})$, $\mathrm{H} / \mathrm{R}+\mathrm{S}$ or $\mathrm{H} / \mathrm{R}+\mathrm{P}$ was generated by the addition of $2 \%$ sevoflurane (Sigma-Aldrich; Merck KGaA) or $50 \mu \mathrm{mol} / 1$ propofol (Sigma-Aldrich; Merck KGaA) to $\mathrm{H} 9 \mathrm{C} 2$ cells cultured in $95 \% \mathrm{O}_{2}$ and $5 \% \mathrm{CO}_{2}$ for $1 \mathrm{~h}$ before being treated as the $\mathrm{H} / \mathrm{R}$ group.

Cell Counting Kit-8 (CCK-8) assay. Cell Counting Kit-8 (TransGen Biotech Co., Ltd.) was used to measure the cell viability, following the manufacturer's instruction. H9C2 cells ( $5 \times 10^{4}$ cells) were inoculated into the well of a 96 -well plate. Before the detection at 1,4 or $7 \mathrm{~h}, 10 \mu \mathrm{l}$ CCK-8 solution was added to the well and incubated in the incubator at $37^{\circ} \mathrm{C}$ for $1 \mathrm{~h}$. After $1 \mathrm{~h}$ of incubation, the absorbance at $450 \mathrm{~nm}$ was detected by a Microplate reader (BioTek Instruments, Inc.).

Bromodeoxynucleoside uracil (BrdU) cell proliferation assay. BrdU Cell Proliferation Assay kit (CST, US) was used to evaluate $\mathrm{H} 9 \mathrm{C} 2$ cell proliferation activity. $\mathrm{H} 9 \mathrm{C} 2$ cells $\left(5 \times 10^{4}\right.$ cells) were inoculated to the well in a 96-well plate. After $48 \mathrm{~h}$ incubation, BrdU solution was added into the 96-well plate and incubated in an incubator for $1 \mathrm{~h}$. Then, the medium was removed, and $100 \mu \mathrm{l}$ fixing/denaturing solution was added into each well for a $30 \mathrm{~min}$ incubation at room temperature. Fixing/denaturing solution was removed, and the sample was incubated with BrdU detection antibody for $1 \mathrm{~h}$. Subsequently, anti-mouse IgG was used to label BrdU detection antibody, and HRP solution was used for coloration. Finally, the absorbance at $450 \mathrm{~nm}$ was detected by a microplate reader (BioTek Instruments, Inc.).

Caspase-3 activity assay. Caspase-3 Activity Assay kit was used to measure H9C2 cell apoptosis rate. After cells were digested by $2.5 \%$ trypsin (Gibco; Thermo Fisher Scientific, Inc.), the lysis solution was used for cell protein extraction, and the protein concentration was at least $1 \mu \mathrm{g} / \mu \mathrm{l}$. The sample (50 $\mu \mathrm{l}$ ) was added to the 96 -well plate with $40 \mu \mathrm{l}$ buffer solution and 10 Ac-DEVD-pNA, and then incubated at $37^{\circ} \mathrm{C}$ for $2 \mathrm{~h}$. After the incubation, the absorbance at $405 \mathrm{~nm}$ was detected by a microplate reader (BioTek Instruments, Inc.).

Total RNA extraction and reverse transcription-quantitative $P C R$ (RT-qPCR). Total RNA was extracted using RNAiso Plus (Takara Bio, Inc.) following standard instructions. The H9C2 cells in a 12-well plate were lysed by $1 \mathrm{ml}$ RNAiso Plus and transferred to a new 2-ml tube without RNase. Then, $200 \mu \mathrm{l}$ chloroform was added to the tube, shaken and mixed. The sample was incubated at room temperature for $5 \mathrm{~min}$ and centrifuged at $10,000 \times \mathrm{g}, 4^{\circ} \mathrm{C}$ for $15 \mathrm{~min}$. The supernatant was transferred to a new $1.5-\mathrm{ml}$ centrifuge tube with $500 \mu \mathrm{l}$ isopropanol. After $10 \mathrm{~min}$ incubation at room temperature, the supernatant was centrifuged at $8,000 \mathrm{xg}$, and $4^{\circ} \mathrm{C}$ for $10 \mathrm{~min}$. Absolute ethanol $(1 \mathrm{ml})$ was used to clean the precipitate and it was subsequently centrifuged at $6,000 \times \mathrm{g}, 4^{\circ} \mathrm{C}$ for $5 \mathrm{~min}$, 
and the supernatant was removed. Finally, $40 \mu \mathrm{l}$ RNase-free was added to the tube to dissolve the precipitate and stored at $-80^{\circ} \mathrm{C}$. ReverTra Ace qPCR RT Master mix with gDNA Remover (Toyobo Life Science) was used for RNA reverse transcription, following the manufacturer's instructions. The relative mRNA expression level was measured by RT-qPCR with QuansStudio 5 instrument (Thermo Fisher Scientific, Inc.). RT-qPCR was performed in three repetitions by using AceQ ${ }^{\circledR}$ Universal SYBR qPCR Master mix (Vazyme Biotech Co., Ltd.) following the manufacturer's instructions. The primers used in RT-qPCR were designed by Oligo 7.0 software (https://www.oligo.net/downloads.html) and synthesized by Sangon Biotech Co., Ltd. The sequences of the primers used in the present study are listed in Table I. Relative mRNA expression was computed using the $2^{-\Delta \Delta \mathrm{Cq}}$ method (21).

Western blotting assay. For protein extraction from cells, ice-cold radioimmunoprecipitation lysis buffer (Beyotime Institute of Biotechnology, Inc.) with $1 \mathrm{mM}$ phenylmethylsulfonyl fluoride (Beyotime Institute of Biotechnology, Inc.) was used. Protein concentration was measured using Pierce ${ }^{\mathrm{TM}}$ Rapid Gold BCA Protein Assay kit (Thermo Fisher Scientific, Inc.). A total of $20 \mu \mathrm{g} /$ lane protein samples were separated by $12 \%$ SDS-PAGE in vertical electrophoresis and transferred onto nitrocellulose membrane using a transfer system (both from BioRad Laboratories, Inc.). After blocking with $5 \%$ skimmed milk for $2 \mathrm{~h}$ at room temperature, the membrane was incubated with anti-ADAM8 (cat. no. ab186432; Abcam) or anti-GAPDH antibody (cat. no. ab9485; Abcam) at $4^{\circ} \mathrm{C}$ overnight in a 1:500 dilution. The anti-rabbit IgG antibody (cat. no. BM2006; Boster Biological Technology) was incubated at room temperature in a 1:10,000 dilution. DAB Horseradish peroxidase color development kit (Beyotime Institute of Biotechnology, Inc.) was used for band imprint display. ImageJ 1.8.0 software (National Institutes of Health) was used for protein quantification.

Dual-luciferase reporter assay. TargetScan was first used to predict what miRNAs might target ADAM8, and then, a dual-luciferase reporter assay was performed to verify the association of the target. In brief, the sequence of ADAM8 3'-UTR with potential target sites of miR-221-5p was synthesized by GeneCreate Biotech and subcloned into pmiR-GLO vector (Promega Corporation), and was named WT. The sequence of ADAM8 3'-UTR was then synthesized, in which potential target sites of miR-221-5p have been mutated, and the mutated sequence was subcloned into the pmiR-GLO vector and was named Mut. Co-transfection was performed in H9C2 cells using Lipofectamine 3000 Transfection Reagent (Thermo Fisher Scientific, Inc.) and the co-transfection groups were: $\mathrm{WT}+$ miR-221-5p mimic, $\mathrm{WT}+$ mimic control, Mut + miR-221-5p mimic, and Mut + mimic control. After $48 \mathrm{~h}$ of co-transfection, firefly and Renilla luciferase activities were measured using a Dual-GLO Luciferase Assay System kit (Promega Corporation) and a microplate reader (BioTek Instruments, Inc.) following the manufacturer's instructions.

Data analysis. Data were analyzed using SPSS 22.0 software (IBM Corp.). One-way ANOVA analysis followed by Dunnett post hoc test was performed for multiple comparisons. An
Table I. Primers' sequences used in the present study.

\begin{tabular}{ll}
\hline Primer & \multicolumn{1}{c}{ Sequences } \\
\hline ADAM8 & F: 5'-AAGCCTACCTCAGGGCTCTC-3' \\
& R: 5'-CTTTGGGGCATAAACAGGAA-3' \\
MicroRNA- & F: 5'-GCCGAGACCTGGCATACAAT-3' \\
221-5p & R: 5'-CTCAACTGGTGTCGTGGA-3' \\
GAPDH & F: 5'-CTCATGACCACAGTCCATGC-3' \\
& R: 5'-TTCAGCTCTGGGATGACCTT-3' \\
U6 & F: 5'-TGCGGGTGCTCGCTTCGGCAGC-3' \\
& R: 5'-CCAGTGCAGGGTCCGAGGT-3'
\end{tabular}

$\mathrm{F}$, forward sequence; $\mathrm{R}$, reverse sequence.

unpaired Student's t-test was applied for comparisons between two groups. ${ }^{*} \mathrm{P}<0.05$ was considered to indicate a statistically significant difference. Data are expressed as mean \pm SD. Each experiment had at least three replicates.

\section{Results}

Sevoflurane exhibits better protective effects than propofol on hypoxia-reoxygenation injury in cardiomyocytes. In order to explore the protective effects of sevoflurane or propofol on hypoxic cardiomyocytes, cell viability, proliferation and apoptosis were detected by CCK- 8 , BrdU and caspase- 3 activity assays. H9C2 cells with no additional treatment were cultured as the control group. On the other hand, after being treated with $\mathrm{H} / \mathrm{R}$ injury, sevoflurane $(\mathrm{H} / \mathrm{R}+\mathrm{S})$ or propofol $(\mathrm{H} / \mathrm{R}+\mathrm{P})$ were added. The CCK-8 assay (Fig. 1A) showed a notable inhibition in the viability of $\mathrm{H} 9 \mathrm{C} 2$ cells treated with $\mathrm{H} / \mathrm{R}$ injury, indicating a severe injury to the cell viability. In spite of the severe injury, both sevoflurane and propofol could restore the effect of $H / R$ injury on cell viability, and sevoflurane had a better effect on the improvement of H9C2 cell injury than propofol. The BrdU assay (Fig. 1B) revealed that the cell proliferation rate was almost $50 \%$ restrained in the $\mathrm{H} / \mathrm{R}$ group compared with that of the control group. Cell proliferation activity in both $\mathrm{H} / \mathrm{R}+\mathrm{S}$ (25\% restrained compared with the control group) and $\mathrm{H} / \mathrm{R}+\mathrm{P}$ group (40\% restrained compared to the control group) was improved, with a better effect in the $H / R+S$ group, again indicating that the effect of sevoflurane was improved compared with propofol in improving H9C2 cell H/R injury. Caspase-3 activity assay (Fig. 1C) was used to determine cell apoptosis, which indicated that $\mathrm{H} / \mathrm{R}$ treatment significantly accelerated the apoptosis of H9C2 cells by nearly three times the normal level. Sevoflurane treatment restored cell apoptosis to a level almost the same as the control group while propofol treatment only restored cell apoptosis to twice the level of that in the control group, indicating that sevoflurane treatment could dramatically compromise the effect of $\mathrm{H} / \mathrm{R}$ injury on cell apoptosis, better than propofol treatment (Fig. 1C). The aforementioned results thus, suggest that cardiomyocytes with H/R injury could be better protected by sevoflurane compared with propofol.

Sevoflurane-rather than propofol-induced ADAM8 upregulation. In order to identify the key genes associated with 
A

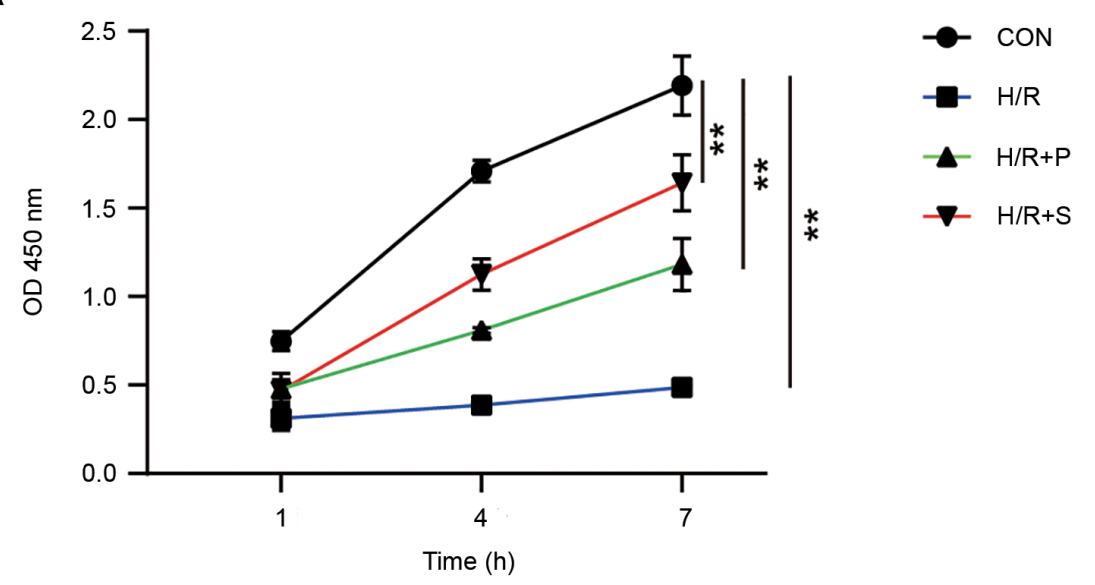

B

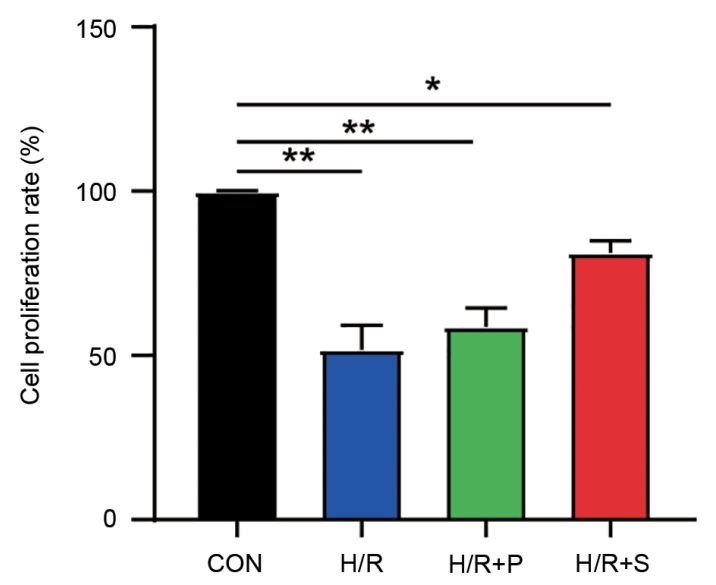

C

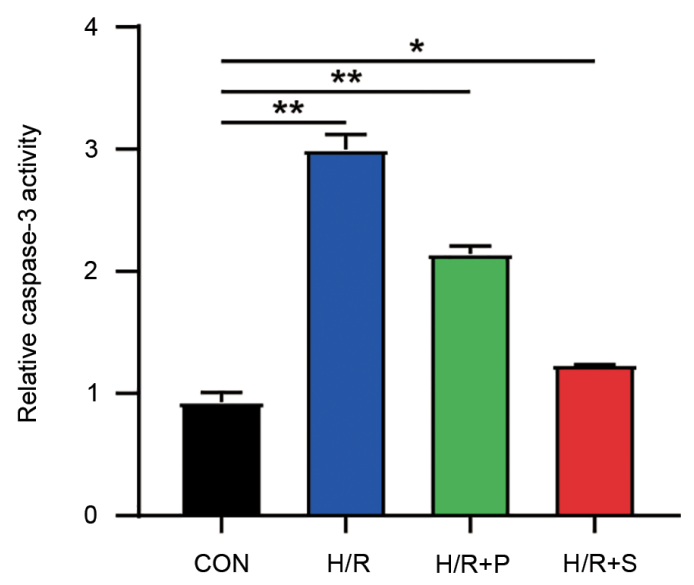

Figure 1. Sevoflurane had improved protective effects than propofol on H/R injury in cardiomyocytes. (A) Cell viability of H9C2 with H/R injury was detected by Cell Counting Kit- 8 assay after treatment with sevoflurane or propofol. (B) Cell proliferation activity was measured by BrdU assay after H9C2 cells with H/R injury were treated with sevoflurane or propofol. (C) Cell apoptosis was determined by caspase-3 activity assay after H9C2 cells with H/R injury were treated with sevoflurane or propofol. Data are presented as mean $\pm \mathrm{SD} .{ }^{*} \mathrm{P}<0.05,{ }^{* *} \mathrm{P}<0.001$. One-way ANOVA was used for the statistical analysis. $\mathrm{H} / \mathrm{R}$, hypoxia-reoxygenation; $\mathrm{CON}$, control; $+\mathrm{P}$, with propofol treatment; $+\mathrm{S}$, with sevoflurane treatment.

sevoflurane and propofol, GSE4386 downloaded from the GEO datasets was used. A total of 37 upregulated genes with $\mathrm{P}<0.05$ and $\log$ fold change $(\mathrm{FC})$ of $>1$ were screened out and uploaded to STRING and Metascape for biological process analysis (Fig. 2A and B). Then, the Venny 2.1.0 analysis result showed that CTSC, CD300A, CLEC4D and ADAM8 were associated with cellular process and the regulation of cell activation (Fig. 2C). A review of the literature revealed that ADAM8 upregulation was associated with I/R injury in rat hearts after cardiac arrest (17), therefore, the function of ADAM8 was explored in cardiomyocytes under $\mathrm{H} / \mathrm{R}$ injury. Next, both the mRNA and protein expression levels of ADAM8 were examined in cardiomyocytes with the treatment of $\mathrm{H} / \mathrm{R}+\mathrm{S}$ and $\mathrm{H} / \mathrm{R}+\mathrm{P}$. The RT-qPCR assay (Fig. 2D) showed that ADAM8 mRNA expression was $\sim 75 \%$ higher in the $\mathrm{H} / \mathrm{R}+\mathrm{S}$ group compared with the $\mathrm{H} / \mathrm{R}+\mathrm{P}$ group. Likewise, western blotting (Fig. 2E) showed that the protein expression of ADAM8 in the $\mathrm{H} / \mathrm{R}+\mathrm{S}$ group was much higher (50\% higher vs. $\mathrm{H} / \mathrm{R}+\mathrm{P}$ group), in agreement with the result of mRNA expression analysis. Taken together, the higher expression of ADAM8 in the $\mathrm{H} / \mathrm{R}+\mathrm{S}$ group indicated that sevoflurane might cause the healing of the H/R injury in cardiomyocytes by co-activating ADAM8.
Sevoflurane exerts improved protective effect than propofol by regulating ADAM8. According to the aforementioned results, ADAM8 could be involved in sevoflurane-mediated healing of H/R injury in cardiomyocytes. Therefore, the association between ADAM8 and sevoflurane was further explored. After successfully transfecting $\mathrm{H} 9 \mathrm{C} 2$ cells with si-ADAM8 and ADAM8-overexpression vector (Fig. S1A), ADAM8 mRNA and protein levels were successfully upregulated by the vector overexpressing ADAM8 and downregulated by si-ADAM8 in $\mathrm{H} 9 \mathrm{C} 2$ cells treated with $\mathrm{H} / \mathrm{R}+\mathrm{S}$ (Fig. $3 \mathrm{~A}$ and $\mathrm{B}$ ). It is suggested that the ADAM8-overexpression vector and si-ADAM8 could successfully transfect $\mathrm{H} 9 \mathrm{C} 2$ cells for use in the subsequent verifications (CCK-8, BrdU and caspase-3 activity assays). CCK-8 assay result (Fig. 3C) showed that ADAM8 overexpression could dramatically increase cell viability while si-ADAM8 decreased cell viability. Likewise, the BrdU assay (Fig. 3D) demonstrated that compared with the $\mathrm{H} / \mathrm{R}+\mathrm{P}$ group, ADAM8 overexpression increases cell proliferation activity by $\sim 75 \%$, while si-ADAM 8 decreased cell proliferation activity by $\sim 60 \%$. Caspase- 3 activity assay was carried out to investigate the effect of ADAM8 on cell apoptosis. The results (Fig. 3E) revealed that ADAM8 overexpression inhibited cell apoptosis by $\sim 70 \%$ relative to the $\mathrm{H} / \mathrm{R}+\mathrm{P}$ group, 
A

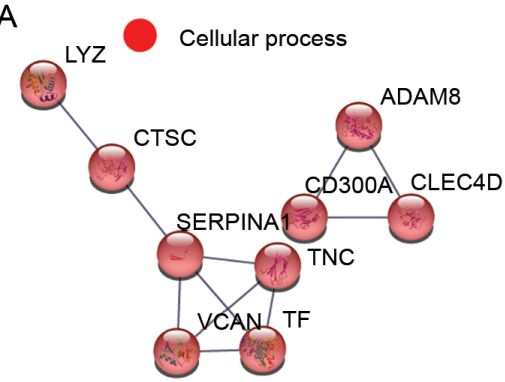

B

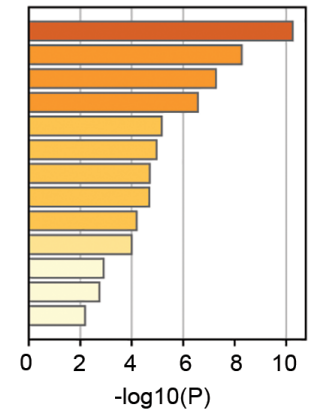

GO:0045055: regulated exocytosis GO:0009617: response to bacterium GO:0002521: leukocyte differentiation GO:0050865 : regulation of cell activation M118: PID INTEGRIN A9B1 PATHWAY GO.0050855: regulation of B cell receptor signaling pathway R-HSA-8057275: Post-translational protein phosphorylation (n) 0:0001894: tissue homeostasis GO:0002250: adaptive immune response 0:0051650: establishment of vesicle localization :0071322: cellular response to carbohydrate stimulus GO:0006875: cellular metalion homeostasis GO:0050900: leukocyte migration

C

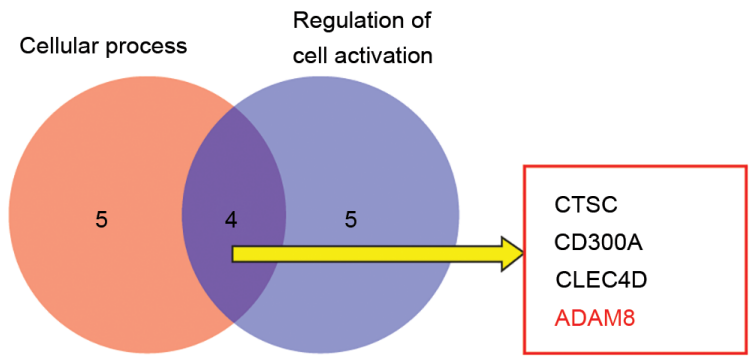

D

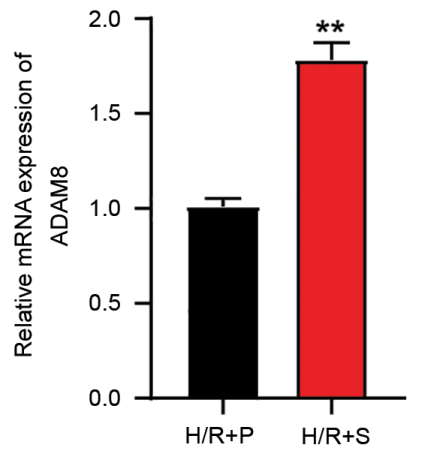

$\mathrm{E}$

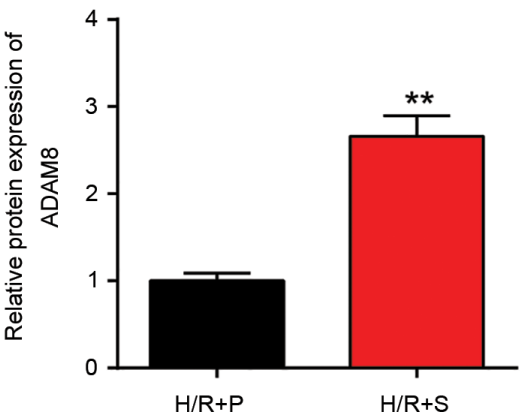

Figure 2. Sevoflurane rather than propofol induces ADAM8 upregulation contributing to the improvement of H/R injury in cardiomyocytes. (A) Key genes were associated with cellular process by STRING analysis. (B) The regulation of cell activation was the key biological process by Metascape analysis. (C) Four genes were overlapped from cellular process and regulation of cell activation. (D) H9C2 cells were subjected to H/R injury and treated with sevoflurane or propofol, and then mRNA expression of ADAM8 was determined by reverse transcription-quantitative PCR assay. (E) H9C2 cells were subjected to H/R injury and treated with sevoflurane or propofol, and then protein expression of ADAM8 was determined by western blotting. Data are presented as mean \pm SD. ${ }^{* *} \mathrm{P}<0.001$. Unpaired t-test was used for the statistical analysis. $\mathrm{H} / \mathrm{R}$, hypoxia-reoxygenation; $+\mathrm{P}$, with propofol treatment; $+\mathrm{S}$, with sevoflurane treatment.

while si-ADAM8 increased the rate of cell apoptosis by more than three-time level in the $\mathrm{H} / \mathrm{R}+\mathrm{P}$ group, and a much higher caspase- 3 activity compared with that in the $\mathrm{H} / \mathrm{R}+\mathrm{P}$ group. The aforementioned results suggest the important role of ADAM8 in sevoflurane-mediated improvement in cardiomyocyte survival from $\mathrm{H} / \mathrm{R}$ injury.

miR-221-5p targets ADAM8 in the regulation of hypoxiareoxygenation injury in cardiomyocytes. The subsequent focus of the study was on the upstream of ADAM8 to further investigate the regulatory mechanisms of ADAM8 in the protection from $\mathrm{H} / \mathrm{R}$ injury in cardiomyocytes and examined the epigenetic regulation of ADAM8 by miRNA. TargetScan was used to identify the potential miRNAs that targeted ADAM8, and the top 4 miRNAs binding to ADAM8 were predicted with Centext score percentile rank (Fig. 4A). The present study focused on exploring the association between miR-221-5p and ADAM8, since the miR-221-5p was the top most-predicted miRNA. The sequence of miR-221-5p matched with the position 274-281 of ADAM8 3'-UTR (Fig. 4B). After detecting the transfection efficiency of the
miR-221-5p mimic and inhibitor (Fig. S1B), dual-luciferase reporter assay was used to verify whether miR-221-5p could bind to ADAM8 3'-UTR. It was shown that the co-transfection of ADAM8 wild type and miR-221-5p mimic could significantly suppress the luciferase activity by $\sim 60 \%$ compared with the control group, whereas ADAM8 mutant type and miR-221-5p mimic failed to decrease the luciferase activity (Fig. 4C), confirming the target association between miR-221-5p and ADAM8 3'-UTR. Given that miR-221-5p could target ADAM8, the expression level of miR-221-5p was assessed in $\mathrm{H} / \mathrm{R}+\mathrm{S}$ - or $\mathrm{H} / \mathrm{R}+\mathrm{P}$-treated $\mathrm{H} 9 \mathrm{C} 2$ cells. The result depicted that the expression of miR-221-5p in the $H / R+S$ group was only $30 \%$ of that in the $\mathrm{H} / \mathrm{R}+\mathrm{P}$ group, which was completely in contrast to the expression of ADAM8 (Fig. 4D). Western blotting assay was performed after the transfections of si-ADAM8, miR-221-5p inhibitor and si-ADAM8 plus miR-221-5p inhibitor. Silencing miR-221-5p could dramatically enhance the protein expression of ADAM8 in cells treated with $\mathrm{H} / \mathrm{R}+\mathrm{S}$ (Fig. 4E). Although si-ADAM8 was capable of decreasing the protein expression of ADAM8, the protein expression level of ADAM8 could be upregulated on 
A

C
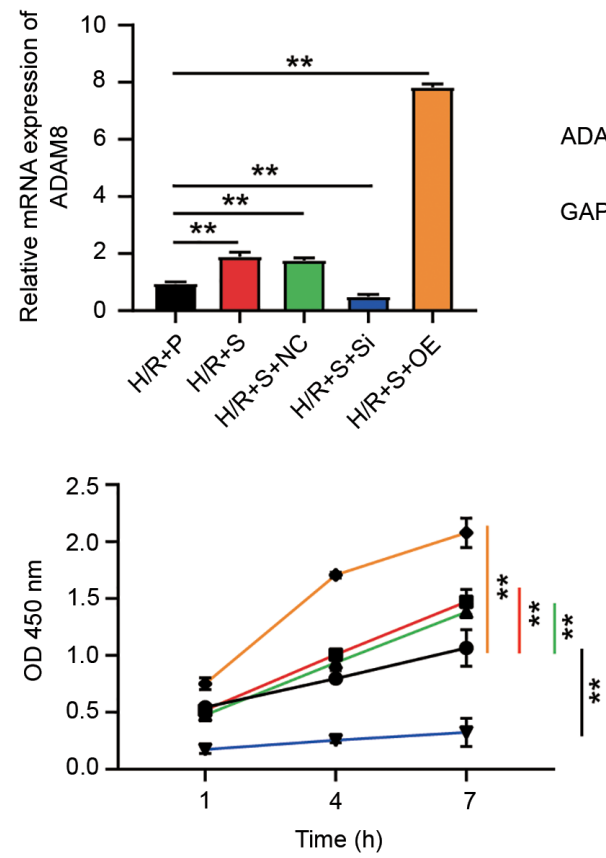

D

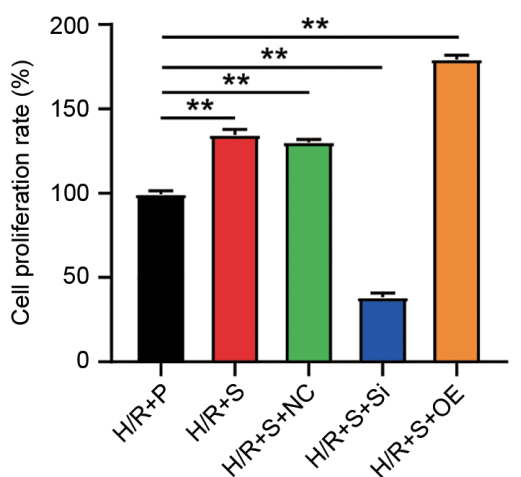

B

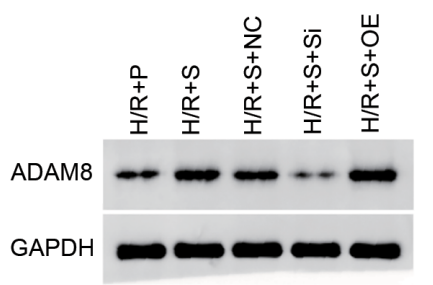

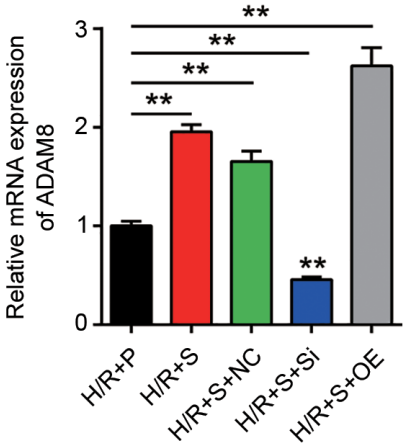

- $H / R+P$

- $H / R+S$

$\simeq H / R+S+N C$

$\rightarrow \mathrm{H} / \mathrm{R}+\mathrm{S}+\mathrm{Si}$

$\bullet \mathrm{H} / \mathrm{R}+\mathrm{S}+\mathrm{OE}$
E

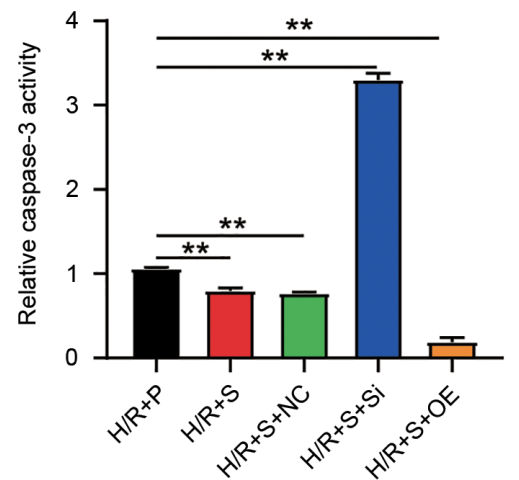

Figure 3. Sevoflurane exerts improved protective effect than propofol by ADAM8 on H/R injury in cardiomyocytes. (A) ADAM8 mRNA expression was detected by reverse transcription-quantitative PCR assay after $\mathrm{H} 9 \mathrm{C} 2$ cells were treated with $\mathrm{H} / \mathrm{R}+\mathrm{P}$, and following transfections with si-ADAM8 or ADAM8-overexpression vector in $\mathrm{H} 9 \mathrm{C} 2$ cells treated with $\mathrm{H} / \mathrm{R}+\mathrm{S}$. (B) ADAM8 protein expression was detected by western blotting assay after H9C2 cells were treated with $\mathrm{H} / \mathrm{R}+\mathrm{P}$ and following transfections with si-ADAM8 or ADAM8-overexpression vector in $\mathrm{H} 9 \mathrm{C} 2$ cells treated with $\mathrm{H} / \mathrm{R}+\mathrm{S}$. (C) Cell viability was detected by Cell Counting Kit-8 assay after H9C2 cells were treated with H/R+P and after transfections with si-ADAM8 or ADAM8-overexpression vector in $\mathrm{H} 9 \mathrm{C} 2$ cells treated with $\mathrm{H} / \mathrm{R}+\mathrm{S}$. (D) Cell proliferation activity was measured by BrdU assay after $\mathrm{H} 9 \mathrm{C} 2$ cells were treated with $\mathrm{H} / \mathrm{R}+\mathrm{P}$ and following the transfections with si-ADAM8 or ADAM8-overexpression vector in $\mathrm{H} 9 \mathrm{C} 2$ cells treated with $\mathrm{H} / \mathrm{R}+\mathrm{S}$. (E) Cell apoptosis was determined by caspase-3 activity assay after $\mathrm{H} 9 \mathrm{C} 2$ cells were treated with $\mathrm{H} / \mathrm{R}+\mathrm{P}$ and after transfections with si-ADAM8 or ADAM8-overexpression vector in $\mathrm{H} 9 \mathrm{C} 2$ cells treated with $\mathrm{H} / \mathrm{R}+\mathrm{S}$. Data are presented as mean $\pm \mathrm{SD},{ }^{* *} \mathrm{P}<0.001$. One-way ANOVA was used for the statistical analysis. $\mathrm{H} / \mathrm{R}$, hypoxia-reoxygenation; $+\mathrm{S}$, with sevoflurane treatment; $+\mathrm{P}$, with propofol treatment; si-, small interfering RNA; NC, negative control; OE, overexpression

inhibiting miR-221-5p. Taken together, sevoflurane is able to decrease miR-221-5p expression and further release the inhibition of ADAM8; thus, playing a role in H/R injury in cardiomyocytes.

Inhibitory effect of miR-221-5p in the protection of hypoxia-reoxygenation injury in cardiomyocytes when treated with sevoflurane or propofol. Since miR-221-5p could target and inhibit the expression level of ADAM8, the activity of $\mathrm{H} 9 \mathrm{C} 2$ cells treated with sevoflurane or propofol was examined after silencing miR-221-5p by an inhibitor. After transfections with si-ADAM8, miR-221-5p inhibitor and si-ADAM8 plus miR-221-5p inhibitor in $\mathrm{H} 9 \mathrm{C} 2$ cells treated with $\mathrm{H} / \mathrm{R}+\mathrm{S}$, respectively, CCK-8, BrdU, and caspase-3 activity assay were conducted. CCK-8 assay (Fig. 5A) showed that miR-221-5p inhibitor could increase cell viability relative to the $\mathrm{H} / \mathrm{R}+\mathrm{P}$ group. However, when miR-221-5p inhibitor and si-ADAM8 were added, cell viability suppressed by si-ADAM8 increased to the same level as that in the $\mathrm{H} / \mathrm{R}+\mathrm{S}+\mathrm{NC}$ group. Similar to the CCK- 8 assay, the BrdU assay also suggested (Fig. 5B) that miR-221-5p inhibitor could increase cell proliferation activity by $\sim 80 \%$ relative to the $\mathrm{H} / \mathrm{R}+\mathrm{P}$ group. However, in the $\mathrm{H} / \mathrm{R}+\mathrm{S}+\mathrm{si}-\mathrm{ADAM} 8+\mathrm{miR}-221-5 \mathrm{p}$ inhibitor group, cell proliferation activity was compromised to a level the same as that in the $\mathrm{H} / \mathrm{R}+\mathrm{S}+\mathrm{NC}$ group. A caspase- 3 activity assay was conducted to explore the effect of the miR-221-5p inhibitor on 
A

\begin{tabular}{lcc}
\hline miRNA & Position in the UTR & Context++ score percentile \\
\hline rno-miR-221-5p & $342-349$ & 99 \\
rno-miR-133c & $51-58$ & 97 \\
rno-miR-877 & $109-116$ & 97 \\
rno-miR-99b-3p & $118-124$ & 97 \\
\hline
\end{tabular}

B

$\begin{array}{ccc}\text { Position 274-281 of ADAM8 3'UTR } & 5, & \text {...UCUGGGUGGACAGAA - - UGCCAGGA... } \\ \text { rno-miR-221-5p } & 3, & \text { C I I I | | | | }\end{array}$

C

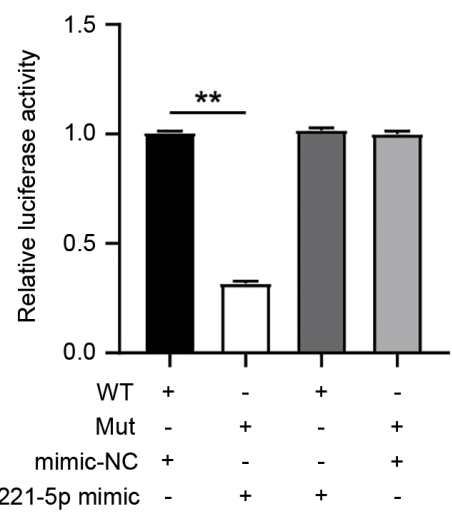

D

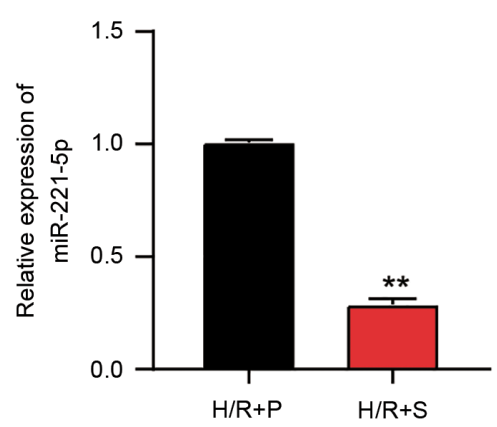

$E$
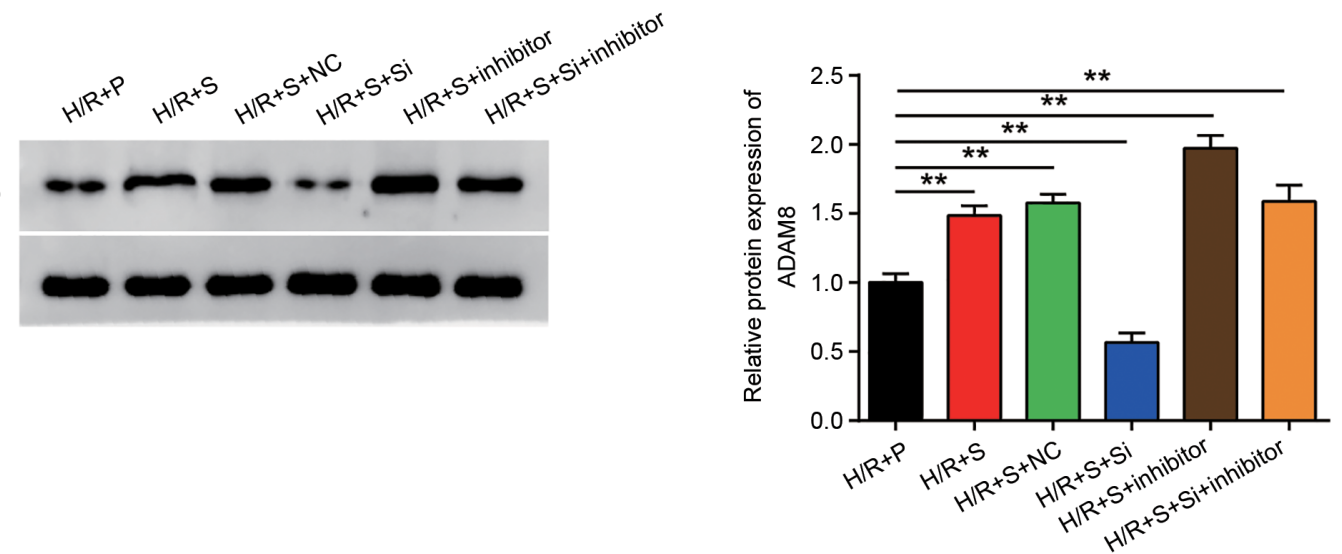

Figure 4. miR-221-5p targets ADAM8 in the regulation of H/R injury in cardiomyocytes. (A) TargetScan was applied to predict the potential miRNAs that might target ADAM8. The top 4 miRNAs are listed. (B) The location to which miR-221-5p binds ADAM8. (C) Dual-luciferase reporter assay was performed to verify the target association between miR-221-5p and ADAM8. One-way ANOVA and Unpaired t-test were used for the statistical analysis. ${ }^{* *} \mathrm{P}<0.001$. (D) $\mathrm{H} 9 \mathrm{C} 2$ cells were subjected to H/R injury and treated with sevoflurane or propofol, and then mRNA expression of miR-221-5p was determined by reverse transcription-quantitative PCR assay. Unpaired t-test was used for the statistical analysis. ${ }^{* *} \mathrm{P}<0.001$. (E) H9C2 cells were subjected to $\mathrm{H} / \mathrm{R}$ injury and treated with sevoflurane or propofol, and then protein expression of miR-221-5p was determined by western blotting assay. One-way ANOVA was used for the statistical analysis. Data are presented as mean $\pm \mathrm{SD}$. ${ }^{* *} \mathrm{P}<0.001$. miR/miRNA, microRNA; H/R, hypoxia-reoxygenation; $+\mathrm{S}$, with sevoflurane treatment; $+\mathrm{P}$, with propofol treatment; NC, negative control; si-, small interfering RNA; WT, wild type; mut, mutant.

cell apoptosis and observed (Fig. 5C) a dramatic inhibition, by $\sim 70 \%$, in cell apoptosis in the miR-221-5p inhibitor group relative to the $\mathrm{H} / \mathrm{R}+\mathrm{P}$ group. In spite of this, the negative effect of the miR-221-5p inhibitor on cell apoptosis could be relieved by si-ADAM 8 co-transfection. These results indicate that the viability of sevoflurane or propofol-treated H9C2 cells can be enhanced by inhibiting miR-221-5p for the protection from $\mathrm{H} / \mathrm{R}$ injury in cardiomyocytes.

\section{Discussion}

Firstly, the effects of anesthetics (sevoflurane and propofol) on cardiomyocytes (H9C2 cells) with H/R injury were investigated and found that sevoflurane had a better protective effect compared with propofol on these cardiomyocytes. Then, the in-depth mechanism underlying the improved cardioprotective effect of sevoflurane on $\mathrm{H} 9 \mathrm{C} 2$ cells exposed to $\mathrm{H} / \mathrm{R}$ injury was explored. miR-221-5p/ADAM8 axis was found to participate in the regulation of sevoflurane in protecting the cardiomyocytes suffering from H/R injury. ADAM8 was found to improve cell activity, while miR-221-5p had an opposite effect on cardiomyocytes with anesthetics treatment and H/R injury. Overall, it was demonstrated that when cardiomyocytes are suffering from H/R injury, sevoflurane is able to regulate the miR-221-5p/ADAM8 axis and exert better protective effects than propofol. 
A

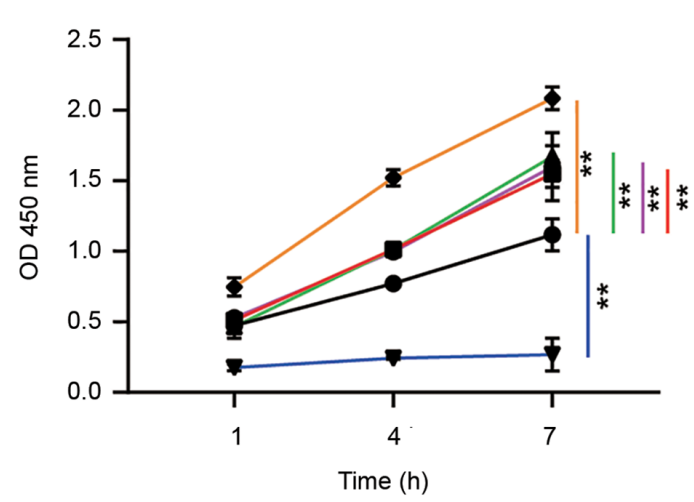

B

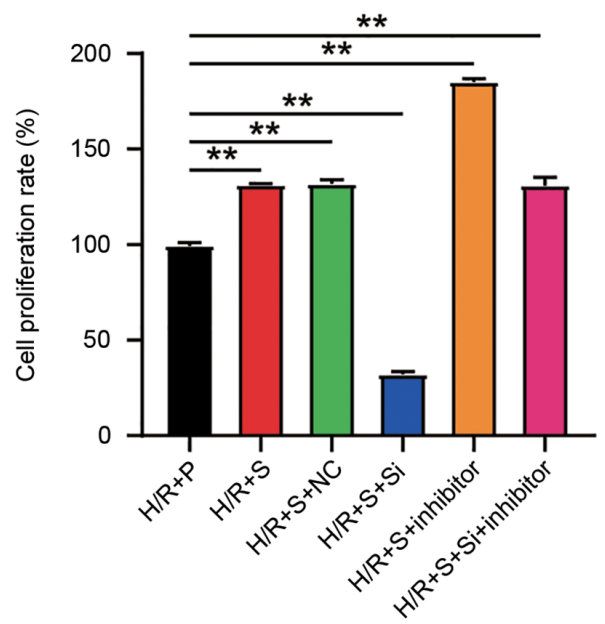

$\rightarrow H / R+P$

$\rightarrow H / R+S$

$-H / R+S+N C$

$\rightarrow \mathrm{H} / \mathrm{R}+\mathrm{S}+\mathrm{Si}$

- $H / R+S+$ inhibitor

- $-H / R+S+S i+i n h i b i t o r$

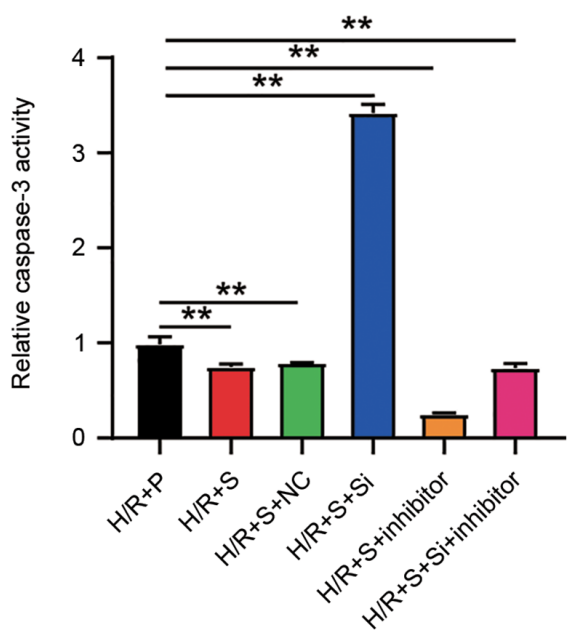

Figure 5. miR-221-5p exhibits an inhibitory effect on the protection of H/R injury in cardiomyocytes treated with sevoflurane or propofol. (A) Cell viability was detected by Cell Cycle Kit- 8 assay after $\mathrm{H} 9 \mathrm{C} 2$ cells were treated with $\mathrm{H} / \mathrm{R}+\mathrm{P}$, and after the transfections with si-ADAM8, miR-221-5p inhibitor or si-ADAM8 plus miR-221-5p inhibitor in $\mathrm{H} 9 \mathrm{C} 2$ cells treated with $\mathrm{H} / \mathrm{R}+\mathrm{S}$, respectively. (B) Cell proliferation activity was measured by BrdU assay after $\mathrm{H} 9 \mathrm{C} 2$ cells were treated with $\mathrm{H} / \mathrm{R}+\mathrm{P}$ and after the transfections with si-ADAM8, miR-221-5p inhibitor or si-ADAM8 plus miR-221-5p inhibitor in H9C2 cells treated with $\mathrm{H} / \mathrm{R}+\mathrm{S}$, respectively. (C) Cell apoptosis was determined by caspase-3 activity assay after $\mathrm{H} 9 \mathrm{C} 2$ cells were treated with $\mathrm{H} / \mathrm{R}+\mathrm{P}$ and after the transfections with si-ADAM8, miR-221-5p inhibitor or si-ADAM8 plus miR-221-5p inhibitor in H9C2 cells treated with H/R+S, respectively. Data are presented as mean \pm SD. ${ }^{* *} \mathrm{P}<0.001$. One-way ANOVA was used for the statistical analysis. H/R, hypoxia-reoxygenation; $+\mathrm{S}$, hypoxia-reoxygenation injury with sevoflurane treatment; $+\mathrm{P}$, hypoxia-reoxygenation injury with propofol treatment; si-, small interfering RNA; NC, negative control.

Until now, multiple studies have shown that sevoflurane is helpful in myocardial protection. For instance, studies have assessed the cardioprotective effect of sevoflurane postconditioning in diabetic rats (22) and the protective role of sevoflurane against the early stage of I/R injury (23). The present study attempted to explore the function of sevoflurane in myocardial $H / R$ cases. Consistent with the previous studies, the present study results provide new evidence of the important role of sevoflurane in protecting cardiomyocytes from I/R injury. It could be confirmed that sevoflurane could protect $\mathrm{H} 9 \mathrm{C} 2$ cells from $\mathrm{H} / \mathrm{R}$ injury by stimulating cell viability and proliferation, while hindering cell apoptosis. In addition to sevoflurane, propofol was also proved to play a protective role in cardiomyocytes in previous studies. Propofol plays a cardioprotective role in myocardial I/R injury through MAPK/ERK signaling pathway (24) and showed a cardioprotective effect on immature hearts at clinically relevant concentrations (25). In the present study, it was also observed that propofol exerted a protective effect on $\mathrm{H} 9 \mathrm{C} 2$ cells exposed to $\mathrm{H} / \mathrm{R}$ injury and that sevoflurane was more protective than propofol in the case of H/R injury in cardiomyocytes. This is in agreement with a previous study (26), which demonstrated that sevoflurane provides greater protection than propofol to the myocardium in patients undergoing off-pump coronary artery bypass surgery and thus provides a reference value in the protection of cardiomyocytes with I/R injury.

ADAM8 was originally identified to be an immune-specific factor (16). In 2019, Schick et al (27) demonstrated the close association of ADAM8 with vascular disease markers, and since then, ADAM8 has been used as an important biomarker in cardiovascular diseases. In the present study, ADAM8 was also determined as a biomarker involved in cardiomyocyte injury. Further investigation revealed that ADAM8 expression is suppressed by propofol treatment (28). Similarly, hypoxia could significantly induce, and propofol could effectively inhibit ADAM8 in another study (29). These two reports are concurrent with the present results that indicated a lower ADAM8 expression level in $\mathrm{H} 9 \mathrm{C} 2$ cells treated with propofol than sevoflurane. This indicated that the better cardioprotective effect of sevoflurane than propofol might rely on the increase in ADAM8 expression level. As expected, the present study results showed that ADAM8 was able to enhance the protective effect of sevoflurane rather than propofol by facilitating cell viability, cell proliferation, and impeding cell apoptosis of H9C2 cells subjected to H/R injury. Another research demonstrated the promotional effect of ADAM8 on cell growth and is in accordance with the present study data showing that knockdown 
of ADAM8 notably inhibited cell proliferation (30). In short, the present study results provide evidence that the prominent protective effect of sevoflurane was promoted by ADAM8 overexpression, which contributed significantly to cell viability and proliferation; thus, enhancing the activity and survival of cardiomyocytes exposed to H/R injury.

To elaborate on the potential factors that might participate in ADAM8-mediated protection of cardiomyocytes with $\mathrm{H} / \mathrm{R}$ injury, the relevant epigenetic mechanism was examined. Using TargetScan and dual-luciferase reporter assay, ADAM8 was predicted and determined as a novel target of miR-221-5p, which exerted a contrasting effect to that of ADAM8. miR-221-5p was earlier found to be expressed at a high level in cardiac hypertrophy and heart failure (20). It was hypothesized that miR-221-5p would attenuate the cardioprotective effect of sevoflurane or propofol in $\mathrm{H} 9 \mathrm{C} 2$ cells subjected to H/R injury. As expected, the present findings collectively suggested that knockdown of miR-221-5p promoted cell viability and proliferation and inhibited cell apoptosis, which could strengthen the protective effect of sevoflurane on cardiomyocytes with heart diseases.

However, the present study did not further investigate the other participants in the downstream signaling pathway of ADAM8. In addition, the present study was restricted to in vitro experiments; in vivo experiments would provide more validation if carried out in the future. Moreover, more clinical analysis and practice are required to verify the present study data and to get a deeper understanding of the mechanisms of sevoflurane, propofol, miR-221-5p and ADAM8 in the recovery and protection of myocardial tissue with H/R injury.

Overall, the present study results show the mechanisms of improved cardioprotective effect by sevoflurane compared with propofol on cardiomyocytes suffering $H / R$ injury through the miR-221-5p/ADAM8 axis. The present study also revealed that sevoflurane protected cardiomyocytes via the upregulation of ADAM8 and downregulation of miR-221-5p. The present study provides novel evidence for the therapeutic strategy against cardiovascular diseases induced by myocardial I/R injury.

\section{Acknowledgements}

Not applicable.

\section{Funding}

No funding was received.

\section{Availability of data and materials}

The datasets used and/or analyzed during the current study are available from the corresponding author on reasonable request.

\section{Authors' contributions}

DX performed the experiments and data analysis. HFD conceived and designed the study. HF designed the methodology. HFD and HF confirm the authenticity of all the raw data. DX wrote the manuscript. HFD reviewed and edited the manuscript. All authors read and approved the final version of the manuscript.

\section{Ethics approval and consent to participate}

Not applicable.

\section{Patient consent for publication}

Not applicable.

\section{Competing interests}

The authors declare that they have no competing interests.

\section{References}

1. Androulakis AE, Andrikopoulos GK, Richter DJ, Tentolouris CA, Avgeropoulou CC, Adamopoulos DA, Toutouzas PK, Trikas AG, Stefanadis CI and Gialafos JE: The role of carotid atherosclerosis in the distinction between ischaemic and non-ischaemic cardiomyopathy. Eur Heart J 21: 919-926, 2000.

2. Li J, Zhang $\mathrm{H}$ and Zhang C: Role of inflammation in the regulation of coronary blood flow in ischemia and reperfusion: Mechanisms and therapeutic implications. J Mol Cell Cardiol 52: 865-872, 2012.

3. Lin Y, Huang S, Chen Y, Wu Z, Liang Z, Zou M and Chen C: Helix B surface peptide protects cardiomyocytes from hypoxia/reoxygenation-induced autophagy through the PI3K/Akt pathway. J Cardiovasc Pharmacol 76: 181-188, 2020.

4. Sun J, Li YZ, Ding YH, Wang J, Geng J, Yang H, Ren J, Tang JY and Gao J: Neuroprotective effects of gallic acid against hypoxia/reoxygenation-induced mitochondrial dysfunctions in vitro and cerebral ischemia/reperfusion injury in vivo. Brain Res 1589: 126-139, 2014.

5. Hwang IC, Kim JY, Kim JH, Lee JE, Seo JY, Lee JW, Park J, Yang HM, Kim SH, Cho HJ and Kim HS: Therapeutic potential of a novel necrosis inhibitor, 7-amino-indole, in myocardial ischemia-reperfusion injury. Hypertension 71 : 1143-1155, 2018.

6. Chen X, Li X, Zhang W, He J, Xu B, Lei B, Wang Z, Cates C, Rousselle $\mathrm{T}$ and Li J: Activation of AMPK inhibits inflammatory response during hypoxia and reoxygenation through modulating JNK-mediated NF- $\mathrm{kB}$ pathway. Metabolism 83: 256-270, 2018.

7. Liang S, Aiqun M, Figtree G and Ping Z: GAPDH-silence preserves $\mathrm{H} 9 \mathrm{C} 2$ cells from acute hypoxia and reoxygenation injury. Int J Biol Macromol 81: 375-386, 2015.

8. Yu J, Wu J, Xie P, Maimaitili Y, Wang J, Xia Z, Gao F, Zhang $\mathrm{X}$ and Zheng $\mathrm{H}$ : Sevoflurane postconditioning attenuates cardiomyocyte hypoxia/reoxygenation injury via restoring mitochondrial morphology. PeerJ 4: e2659, 2016.

9. Cope DK, Impastato WK, Cohen MV and Downey JM: Volatile anesthetics protect the ischemic rabbit myocardium from infarction. Anesthesiology 86: 699-709, 1997.

10. Zhang Y, Chen Z, Feng N, Tang J, Zhao X, Liu C, Xu H and Zhang M: Protective effect of propofol preconditioning on ischemia-reperfusion injury in human hepatocyte. J Thorac Dis 9: 702-710, 2017.

11. Navapurkar VU, Skepper JN, Jones JG and Menon DK: Propofol preserves the viability of isolated rat hepatocyte suspensions under an oxidant stress. Anesth Analg 87: 1152-1157, 1998.

12. Li YC, Ridefelt P, Wiklund $\mathrm{L}$ and Bjerneroth G: Propofol induces a lowering of free cytosolic calcium in myocardial cells. Acta Anaesthesiol Scand 41: 633-638, 1997.

13. Lu Y,Bu Mand Yun H: Sevoflurane prevents hypoxia/reoxygenationinduced cardiomyocyte apoptosis by inhibiting PI3KC3-mediated autophagy. Hum Cell 32: 150-159, 2019.

14. Takahashi Y, Shibata T, Sasaki Y, Hirai H, Takemura S, Minamiyama Y, Sakaguchi M and Suehiro S: Pre-ischemic administration of landiolol prevents ischemia-reperfusion injury in the rat heart. Osaka City Med J 53: 9-16, 2007.

15. Wolfsberg TG, Straight PD, Gerena RL, Huovila AP, Primakoff P, Myles DG and White JM: ADAM, a widely distributed and developmentally regulated gene family encoding membrane proteins with a disintegrin and metalloprotease domain. Dev Biol 169: 378-383, 1995. 
16. Yoshiyama K, Higuchi Y, Kataoka M, Matsuura K and Yamamoto S: CD156 (human ADAM8): Expression, primary amino acid sequence, and gene location. Genomics 41: 56-62, 1997.

17. Vuohelainen V, Raitoharju E, Levula M, Lehtimaki T, Pelto-Huikko M, Honkanen T, Huovila A, Paavonen T, Tarkka M and Mennander A: Myocardial infarction induces early increased remote ADAM8 expression of rat hearts after cardiac arrest. Scand J Clin Lab Invest 71: 553-562, 2011.

18. Liu J, Wang J, Ning Y and Chen F: The inhibition of miR-101a-3p alleviates $\mathrm{H} / \mathrm{R}$ injury in $\mathrm{H} 9 \mathrm{C} 2$ cells by regulating the JAK2/STAT3 pathway. Mol Med Rep 21: 89-96, 2020.

19. Huang P, Lin Q and Liu X: MicroRNA-142-5p inhibits autophagy in cardiomyocytes in a mouse model of hypoxia/reoxygenation. FEBS Open Bio: May 11, 2020 (Epub ahead of print).

20. Shah R, Ziegler O, Yeri A, Liu X, Murthy V, Rabideau D, Xiao CY, Hanspers K, Belcher A, Tackett M, et al: MicroRNAs associated with reverse left ventricular remodeling in humans identify pathways of heart failure progression. Circ Heart Fail 11: e004278, 2018.

21. Livak KJ and Schmittgen TD: Analysis of relative gene expression data using real-time quantitative PCR and the 2(-Delta Delta C(T)) method. Methods 25: 402-408, 2001.

22. Xie P, Yang L, Talaiti A, Wu JJ, Yu J, Yu T, Wang HY, Huang B, Wu Q, Maimaitili Y, et al: Deferoxamine-activated hypoxia-inducible factor-1 restores cardioprotective effects of sevoflurane postconditioning in diabetic rats. Acta Physiol (Oxf) 221: 98-114, 2017

23. Ohsumi A, Marseu K, Slinger P, McRae K, Kim H, Guan Z, Hwang DM, Liu M, Keshavjee S and Cypel M: Sevoflurane attenuates ischemia-reperfusion injury in a rat lung transplantation model. Ann Thorac Surg 103: 1578-1586, 2017.
24. Yan HJ, Qi GQ and Ma Y: Effect of propofol on myocardial ischemia-reperfusion injury through MAPK/ERK pathway. Eur Rev Med Pharmacol Sci 23: 11051-11061, 2019.

25. Shirakawa M, Imura H and Nitta T: Propofol protects the immature rabbit heart against ischemia and reperfusion injury: Impact on functional recovery and histopathological changes. Biomed Res Int 2014: 601250, 2014.

26. Conzen PF, Fischer S, Detter C and Peter K: Sevoflurane provides greater protection of the myocardium than propofol in patients undergoing off-pump coronary artery bypass surgery. Anesthesiology 99: 826-833, 2003.

27. Schick D, Babendreyer A, Wozniak J, Awan T, Noels H, Liehn E, Bartsch JW, Vlacil AK, Grote K, Zayat R, et al: Elevated expression of the metalloproteinase ADAM8 associates with vascular diseases in mice and humans. Atherosclerosis 286: 163-171, 2019.

28. Yu X, Shi J, Wang X and Zhang F: Propofol affects the growth and metastasis of pancreatic cancer via ADAM8. Pharmacol Rep 72: 418-426, 2020.

29. Gao Y, Yu X, Zhang F and Dai J: Propofol inhibits pancreatic cancer progress under hypoxia via ADAM8. J Hepatobiliary Pancreat Sci 26: 219-226, 2019.

30. Yang Z, Bai Y, Huo L, Chen H, Huang J, Li J, Fan X, Yang Z, Wang L and Wang J: Expression of A disintegrin and metalloprotease 8 is associated with cell growth and poor survival in colorectal cancer. BMC Cancer 14: 568, 2014.

This work is licensed under a Creative Commons Attribution-NonCommercial-NoDerivatives 4.0 International (CC BY-NC-ND 4.0) License. 